\title{
How much help do repeated presentations give to recognition processes?'
}

\author{
MARVIN DAINOFF AND RALPH NORMAN HABER
}

UNIVERSITY OF ROCHESTER

A recent experiment by Haber and Hershens on (1965) had shown that in a recognition task one long look at a stimulus was always superior to two or more shorter looks surming to the same total presentation time. In order to explore this more fully and to account for opposite results in a different type of recognition task, as well as in a serial learning task, an improved replication of the earlier study was carried out using very short durations and single letiers as stimuli. The same non-reciprocity was found, again strongly favoring duration over repetition as a determinant of clarity of a percept, even though repetition alone was also shown to be a significant independent variable. As a subsidiary finding, an error analysis showed that when a letter was misnamed it was nearly always confused with one that looked like it rather than one that sounded like it. Some discussion was offered as to the role of an auditory information storage in low memory load tasks such as this one, as well as some general implications for information processing analyses of the nonreciprocity of duration and repetition.

Research on temporal integration (e.g., Boynton, 1961) has suggested that within the integration period, it does not matter how presentations are distributed in time. Thus, two $10-\mathrm{msec}$. flashes are equivalent to one $20-\mathrm{msec}$. flash, and further, the separation between the two $10-\mathrm{msec}$. flashes is irrelevant as long as the total time from onset of the first to offset of the second does not exceed the integration constant. The value of the integration constant depends, of course, upon the relative luminances of the target and adaptation fields, as well as on the task of the perceiver (absolute threshold, judgment, discrimination of successiveness, brightness matching), but generally the upper limit is around $100 \mathrm{msec}$. Within this time then it is possible to chop up the total energy package and redistribute it helter-skelter in time without affecting the perceiver's responses to it.

A similar kind of investigation can be pursued about a seemingly unrelated problem. Assume an energy package of fixed luminance, and of a duration of 50 msec., for example. Is the perceiver equally as efficient in recognizing the content of the stimulus in one presentation of $50 \mathrm{msec}$, as in two well separated (e.g., one-to-ten sec. apart) presentations of $25 \mathrm{msec}$., as in four presentations of 12-1/2 msec., etc.? Since these interstimulus intervals are several orders of magnitude larger than the integration constant, the answer would seem to be "no."

However, some recent data showing the effect of repetition on word and letter recognition suggest that perhaps the "no" may be too hasty. A series of studies by Haber and Hershenson (1965), Hershenson and Haber (1965), Haber (1965), Haber and Hillman (1966) and Hershenson (1965), have shown that if words or letters are presented for from one to 25 times, with no change in duration or luminance, the clarity of the letters increases dramatically as a function of the number of repetitions. Thus, holding energy constant, the clarity of the letters of the stimulus increases with repetitions alone, even when the repetitions are spaced as much as 10 sec. apart. This effect is found with frequent English words, with Turkish words, with rare English words, with rare and frequent English words for which the subject is clearly shown each word just prior to its first presentation, with single English letters, and with three and four letter nonsense words. Further, in each case, the rate of increase in clarity of the letters of the stimuli follows the same mathematical function, indicating that the effect of repetition is independent of the structure and meaning of the stimulus. (See Haber, 1966a, for a summary of the earlier of these studies, and Haber, $1966 \mathrm{~b}$, for a more detailed discussion.)

The relevance of these studies comes from the fact that repetition will increase the clarity of an item of the stimulus without changes in the intensity, duration, or other stimulus characteristics. Therefore, one can ask whether a reciprocity exists between these energy variables and repetition. Will the perceiver perform as well with one flash of $50 \mathrm{msec}$. as he will with two of $25 \mathrm{msec} . ?$ A $50 \mathrm{msec}$. flash will lead to better performance than a $25 \mathrm{msec}$. one, and also two flashes will lead to better performance than one flash. What is the relationship between these two independent variables?

In their first experiment, Haber and Hershenson (1965) presented some data to suggest that duration is always a more powerful variable than repetition. For every combination tested, one long flash always resulted in greater clarity than two or more shorter flashes summing to the same total time. They presented this evidence somewhat tentatively because their variation in duration was quite coarse. Thus, they might have found two flashes at $10 \mathrm{msec}$. to be poorer than one at $20 \mathrm{msec}$. because the increase of $10 \mathrm{msec}$. was large enough to overwhelm the changes produced by adding an additional repetition. With this possibility in mind, the reciprocity issue was dropped.

Gilinsky (1964) reported some data drawing the opposite conclusions from a different kind of experiment. Using a modified method of limits, she presented rows of digits to be recognized, and found, as 
had the experiments just cited, that holding duration constant, Ss were more accurate the more repetitions they had. However, when she varied duration and repetition together, in order to plot reciprocity curves, she found that accuracy was more a function of repetition than of duration. Thus, it was easier to correctly recognize the items in the display from two flashes of $25 \mathrm{msec}$, than from one flash of $50 \mathrm{msec}$. This was true for each of the array sizes she used.

The most likely explanation for this discrepancy in results concerns Gilinsky's use of the method of limits, which permitted the perceiver as many flashes as he needed to achieve a correct response, since he could choose to report or not on each flash. In the Haber and Hershenson study and, in fact, in all of the others as well, the number of flashes was determined beforehand, so that the S's performance could not change or affect it. In this way, the $S$ had to report all that he knew on each flash, since he could not assume that he could have another opportunity. Gilinsky's use of the method of limits, as does everyone else's, confounds the effect of repetition with the perceiver's confidence in his report and his expectations about how many flashes he will have. The likely direction of the confounding here would be to exaggerate the variance due to repetition, which is consistent with what Gilinsky found.

There seems to be no other directly relevant evidence on this question. Several studies have given an $S$ either one or two presentations of a stimulus and then asked him to describe, identify, recognize or detect it (e.g., Eriksen \& Hoffman, 1963; Pylyshyn, 1965; Smith \& Carey, 1966). While each of these finds the multi-presentation superior, supporting the repetition effects reported earlier, only the design of Smith and Carey (1966) permits a reciprocity analysis between repetition and some energy parameter of the stimulus, and their interpresentation interval was so brief it might have been within an integration constant.

There is also some literature in serial learning that is of indirect relevance. For example, Mayzner and Schoenberg (1965) have shown that the presentation time for each item to be learned is reciprocally related to the number of times it is presented. Thus, four repetitions of a list at $1 \mathrm{sec}$. per item yields an equivalent learning performance as one repetition at $4 \mathrm{sec}$. per item. This is mentioned because the reciprocity between duration and repetition is similar to that under discussion. However, the task for the $\mathrm{S}$ is quite different (memorization under high memory load versus recognition under low memory load). The mechanisms must also be quite different-the former probably indicating that time available for rehearsal is the relevant process, while some other as yet unknown process underlies the repetition effect in recognition.

One common thread running through all of this research is relevant to considerations of information processing. The question can be asked: What is the relationship between the energy (intensity and duration) needed for adequate registration of stimulation, and the processes that then occur which are designed to solve the problem of what the stimulus was, or to make decisions based on its identity, or to commit it to memory? (See Haber, 1967, for a detailed discussion of this problem). In this context, does extending the time the stimulus is presented enable the perceiver to perform these post-registration tasks more effectively, as compared to giving him a second independent opportunity to do it? Some theorists have argued (e.g.. Sperling, 1960) that the adequacy of the short term visual storage set up by a stimulus is independent (within broad limits) of the presentation duration of the stimulus. If this is the case, then having two or more separate opportunities to process that short term visual storage should produce greater accuracy on the criterion tasks than only one opportunity, assuming that the short term visual store is equally adequate in each case. This assumption may be a difficult one to accept, even granting Sperling's data (1960). It needs to be tested much more extensively.

With all of these issues somehow in mind, another look was taken at this problem for a recognition task. To repair the major difficulty in the Haber and Hershenson study, a much shorter duration value was used so the changes in duration would be quite small in relation to repetition changes. Rather than increase luminance levels, the stimulus was simplified, so that instead of using seven letter words, as in the earlier study, in this experiment single letters were used, being much simpler and also falling completely within the fovea. Pretesting showed that Ss can easily see a single letter at 5 to $10 \mathrm{msec}$. with one flash, and could see one at 2 to $4 \mathrm{msec}$. If given several flashes. Other than this change, the procedure was basically drawn from the Haber and Hershenson experiment.

\section{Subjects}

\section{METHOO}

Three adults with normal corrected vision who had at least $4 \mathrm{hr}$. of practice under experimental conditions served as Ss.

\section{Apparatus and stimulus materials.}

Stimuli were single letters of the alphabet, individually drawn in black ink on white $4 \times 5$ cards using a LeRoy lettering stencil No. 3240-200CL and pen No. 3233. Each letter was $3 / 16$ in. high-subtending $20 \mathrm{~min}$. of visual arc vertically-and varied from $1 / 32$ in. to $3 / 16$ in. in width. Contrast ratio was $98 \%$. Twentyfive of the 26 letters of the alphabet were used; " $Q$ " being eliminated. Stimuli were presented in the stimulus channel of a two channel mirror tachistoscope (Scientific Prototype Type 800F). The second channel 
served as background and contained two horizontal párallel fixation lines which were spaced $1 / 8$ in. above and below the stimulus. The background channel remained lighted except when a stimulus was being presented in the first channel. Each channel was set at the beginning of each session to a luminance of 20 ft.-L, measured with a Gamma Scientific photometer (Model 700M). (For the first several sessions, values of 10,15 , and $25 \mathrm{ft}$.-L were sometimes used because of equipment problems; however, the luminances in each channel were always equal. There was no apparent effect of this procedural variation in the data.) Following each experimental session, luminance readings were again taken to insure that both channels had remained at a constant luminance.

\section{Procedure}

Each S was run for 15 daily 1-hr. sessions, after at least $4 \mathrm{hr}$. previous training on the experimental task (S JD had $12 \mathrm{hr}$. of pilot experience). Each daily session consisted of four duration (D) by repetition (N) conditions such that $\mathrm{D} \times \mathrm{N}=\mathrm{k}$ for each condition. There were 15 stimuli per condition or 60 stimuli per daily session. The number of trials (N) for the four conditions was always $3,4,5$, and 6 ; with accompanying values of duration varying each day as a function of daily threshold. (Threshold estima tion procedures are described below.) Stimulus letters were randomized across sessions such that each letter appeared an equal number of times at each (D $x$ N) condition. The sequence of appearance of conditions within each session was likewise randomized. Each $S$, therefore, saw 225 stimuli per condition across the 15 sessions, so that the (reduced) alphabet was randomly repeated five times.

The $\mathrm{S}$ presented the stimulus to himself by use of a push button upon command of $\mathbf{E}$. During a typical sequence, $S$ would press to see the stimulus, report what letter he saw, if any, and immediately be instructed again to press the button. Alternate pressing and reporting continued until 8,9 , or 10 trials occurred; $\mathrm{E}$ then informed $\mathrm{S}$ of the identity of the stimulus, and changed the stimulus card. Interflash intervals ranged from 1 to 1.5 sec. Decks of stimulus cards were arranged beforehand and inserted into the apparatus, and were removed individually after each was viewed by $\mathrm{S}$. The $\mathrm{E}$ was, therefore, ignorant of the identity of the stimulus until after $S$ had finished viewing it. It is to be noted that, although $S$ saw each stimulus 8 to 10 times, only the Nth trial, corresponding to one of the four $\mathbf{D} \times \mathbf{N}$ conditions was scored. Thus, if, for example, a given stimulus is assigned to a condition of $3 \mathrm{msec}$. $x 6$ trials, $\mathrm{S}$ may actually be presented the letter for 10 trials at $3 \mathrm{msec}$. each, but only the sixth trial is scored. (This variation was included to attempt to eliminate an end point effect discussed by Haber and Hillman, 1966, and Hershenson, 1965 in which Ss showed an unexplained but distinct tendency to be less correct on the last trial.)

The Ss were explicitly instructed not to guess, but to report exactly what they saw. A problem solving set was discouraged. The $S$ was further informed that he could not predict either the next letter or the number of trials he would get. Needless to say, $S$ was given no idea that only certain trials were being scored.

The procedure used in obtaining four stimulus duration values required estimation of the daily threshold for each $S$. To do this, it was decided that one of the four durations would be above threshold, one at threshold, and two below. To estimate the threshold at the beginning of each session, 27 stimuli were shown to $S$ under the same presentation conditions as described earlier, except that he received 6,7 , or 8 trials per stimulus, and for these determinations, only the fourth trial was scored. A modified staircase method was used in which a correct response on the fourth trial for a particular letter produced a decrease of $0.5 \mathrm{msec}$. in the exposure duration for the next stimulus; an incorrect response on trial four led to an 0.5 msec. increase for the next stimulus. This was continued for 27 stimuli. Then $S$ had a rest period while $E$ prepared a plot of frequency correct vs. duration, and determined the $50 \%$ correct point as the threshold $(T)$ to the nearest $.01 \mathrm{msec}$. Since $T$ was based on trial four, $T$ became the duration value for the condition in which $\mathrm{N}=4$ for that day. For example, if $T$ was $3.00 \mathrm{msec}$. for four flashes, then the total exposure time over the four trials is 12 msec. Thus, the exposure duration of each of the flashes, when five flashes are given, is given by $12 / 5=2.5$ msec., and similarly for the three flash and six flash conditions. In this way for all $D \times N$ conditions, the total time the stimulus is on view is held at a constant. That constant varied slightly from day to day as a function of threshold variations.

\section{RESULTS}

Examination of the daily thresholds for each $S$ gave no indication of any systematic trend across sessions; variability within and between Ss was low. The mean threshold for each $\mathrm{S}$ was $2.91,3.14$, and 3.33 msec. respectively, with standard deviations across sessions of $.31, .46$, and .42 respectively. While the mean threshold values are indicated on the graphs, actual data points are based on a simple combination of percent of number correct per condition for each day across days.

Figure 1 indicates for each $S$, the number of stimuli correctly reported on the Nth trial of each of the four $D \times N$ conditions. These results may be compared with hypothetical functions indicating three possible extreme outcomes: effect of duration only, repetition only, or reciprocity. There is little question that duration effects are clearly superior to repetition 

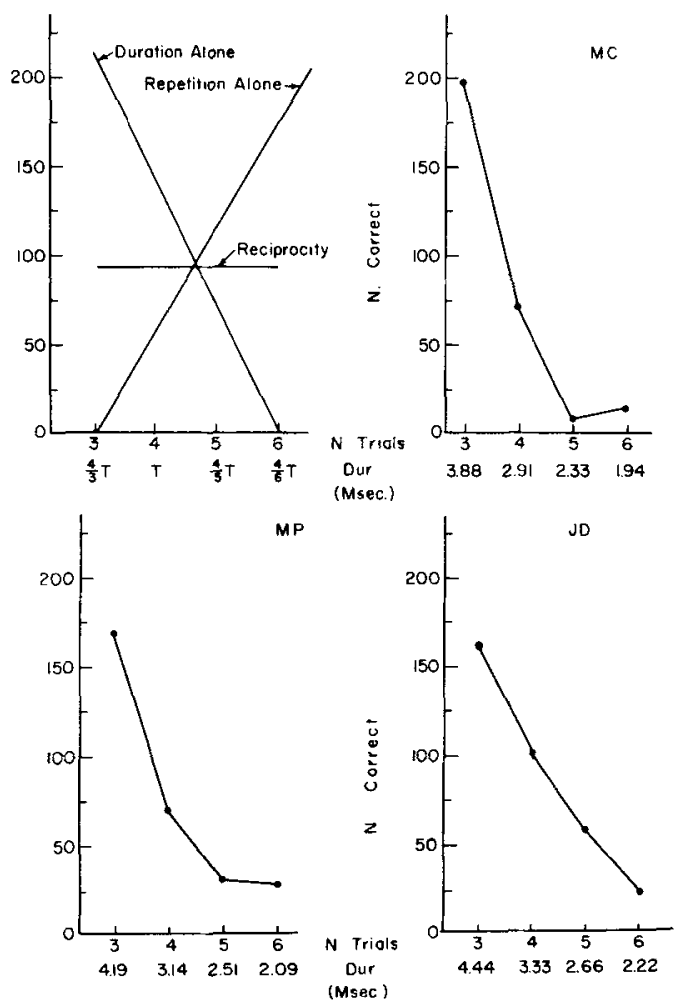

Fig. 1. Number of correct responses on the $N$ th trial for four experimental conditions, where $D \times N=k$ for each condition. Results for three Ss can be compared with hypothetical functions illustrating extreme cases of possible altemative effects.

effects, and the postulation of a reciprocity relationship is untenable. The lack of change between trials 5 and 6 for Ss MC and MP is almost certainly a lower limit on performance rather than a reciprocity.

Figure 2 indicates an attempt to examine the data in a manner comparable to that of Haber and Hershenson (1965) and Haber and Hillman (1966), so that growth of a percept as a function of duration alone can be seen. In this analysis, percent correct for each trial, for trials 1-9, was plotted for each experimental condition. Trial 10, having many fewer points than the others, was eliminated. (The value for $\mathrm{N}$ in the parameter is, of course, irrelevant here and serves only for identification.) It can be seen that, at least for the two longest durations, percent correct increases over trials. Thus, growth as a function of repetition alone, with duration held constant, can be inferred. The portion of the threshold curvecondition ( $4 \times \mathrm{X}$ )-over trials $1-5$ can be considered a direct replication of Haber and Hillman (1966) since that was the only condition they ran, and these partial curves do, in fact, superimpose themselves well within the limits of the Haber and Hillman curves.

Returning to the cases in which only the Nth trial is scored, an attempt was made to provide a converging operation on the state of the information-

processing sequence at that point in time. Accordingly, responses on trial $\mathrm{N}$ were categorized as to whether the letter was correctly reported, some other letter was reported (an error), or nothing was reported. Percentages of these categories are plotted in Fig. 3 as a function of experimental condition. An interesting result is that while the percent of correct responses and the percent of nothing seen responses are mirror images of each other, the rate of error stays fairly constant, although at different levels for each $\mathrm{S}$. To examine this further, a more molecular analysis of the errors was made by considering just those Nth trial responses shown in Fig. 3 on which responses were incorrect. A classification of type of error was established as: visual confusion (e.g., D confused with L); auditory confusion (e.g., $B$ with $V$ ); visual and/or auditory confusion (e.g., B with D); or as an unclassifiable confusion (e.g., $X$ with $O$ ). A criterion for making the classification was provided by preparation of a confusion matrix, in which each of the 25 letters was paired with every other one. Three judges indicated into which of the four classifications each of the pairs $(N=300)$ fell. The reliability of the judges' decision was indicated by their having fewer than $15 \%$ initial disagreements, each of which were easily resolved by discussion. The matrix was then used for scoring the error responses. Generation of the confusion matrix indicated that the a priori probabilities of the four types of errors within the matrix given one repetition of the 25-letter alphabet were: $\mathrm{Pv}=.32 ; \mathrm{Pa}=.07 ; \mathrm{Pb}=.08$; and $\mathrm{Pu}=.53$ respectively. These values were then used to correct raw percentages of each type of error using Bayes Theorem. For example, consider one $S$ (MP) at condition ( $3 \times 4.19)$. According to Fig. 3, $19 \%$ or 42 of the 225 responses in that condition were
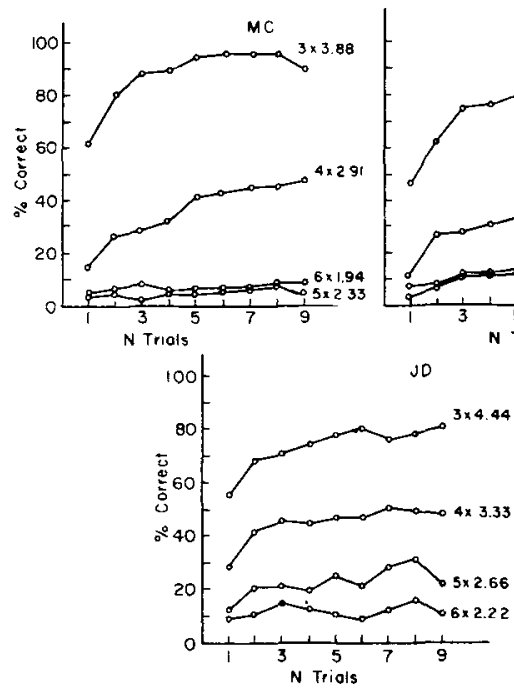

Fig. 2. Per cent of correct responses as a function of the number of trials, for four durations, for each of three Ss. 


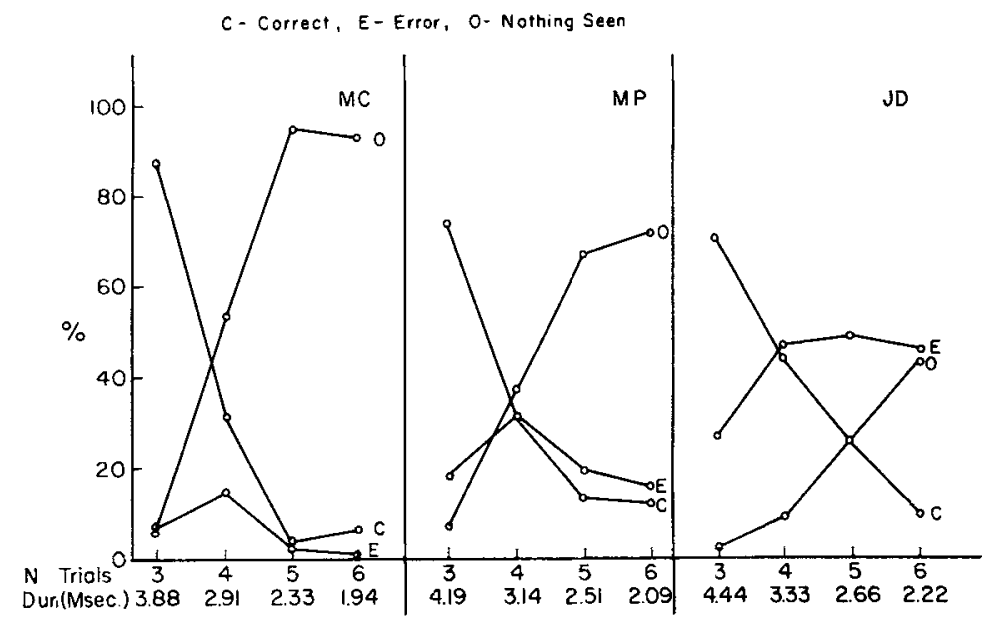

Fig. 3. Per cent of responses that were correct $(C)$; in error $(E)$; or nothing seen $(O)$, for three $\mathrm{Ss}$ on the $\mathrm{N}$ th trial, for four experimental conditions.

errors. Of these 42 errors, 32 were of the visual type. However, since the a priori probability of making a visual error is .32 , the number of expected visual errors for any condition is $(.32 \times 225)$ or 73 . The proportion of observed-to-expected visual errors for this condition is $32 / 73$ or .44. Similar observed-toexpected proportions for $A, B$, and $U$ type errors are .06, .33, and .02. These four proportions are summed to .86 , and the corrected probability of a visual error is $.44 / .86$ or .51 .

Figure 4 indicates these corrected probabilities of making a given type of error for each $\mathrm{S}$ in each condition. While the profiles of the individual Ss are not perfectly consistent, it is clear that pure auditory errors and unclassified errors are rare for all Ss in all conditions, and that visual errors and errors that cannot, in this experiment, be differentiated between visual and auditory confusions, predominate. Since Kaplan, Yonas, and Shurcliff (1966) found in a related task that visual confusions accounted for the errors in this mixed category, it seems virtually certain that visual confusions underlie nearly all or the errors made in this task.

\section{DISCUSSION}

The lack of a reciprocity effect supports the earlier Haber and Hershenson (1965) study. The magnitude of the duration change seems to be unimportant, for even when duration is changed by less than $1 \mathrm{msec}$, changing repetition cannot counteract this effect on performance. Thus, even though quite high levels of performance can be attained in an absolute sense by increasing repetitions, giving $S$ a longer look is always better than giving him several shorter looks.

The discrepancy between this and the Gilinsky study still remains, reinforced somewhat by this replication. With respect to the implications regarding Sperling's model, either the assumption that the duration of $3 \mathrm{msec}$. creates an equivalent short term visual storage as one of $4 \mathrm{msec}$. is wrong, or it is necessary to postulate that a longer stimulation facilitates recognition processes over and above an increased adequacy of a short term visual storage. Since the present experiment was obviously not designed to explore these questions beyond the intimations given, further badly needed rasearch will have to settle this issue.

The error analysis (Fig. 3) shows that the rate of making errors (as compared to the rate of making correct responses or of saying nothing was seen) was fairly independent of duration and repetition. On the assumption that the error of misnaming a letter is analogous to a false alarm rate, this shows that the false alarm rate is somewhat invariant over repetitions, a finding supporting the argument that the gain in clarity with repetition is not due to $S$ lowering his criterion and being more willing to guess. This has also been shown by Haber and Meiselman (1967) more directly.

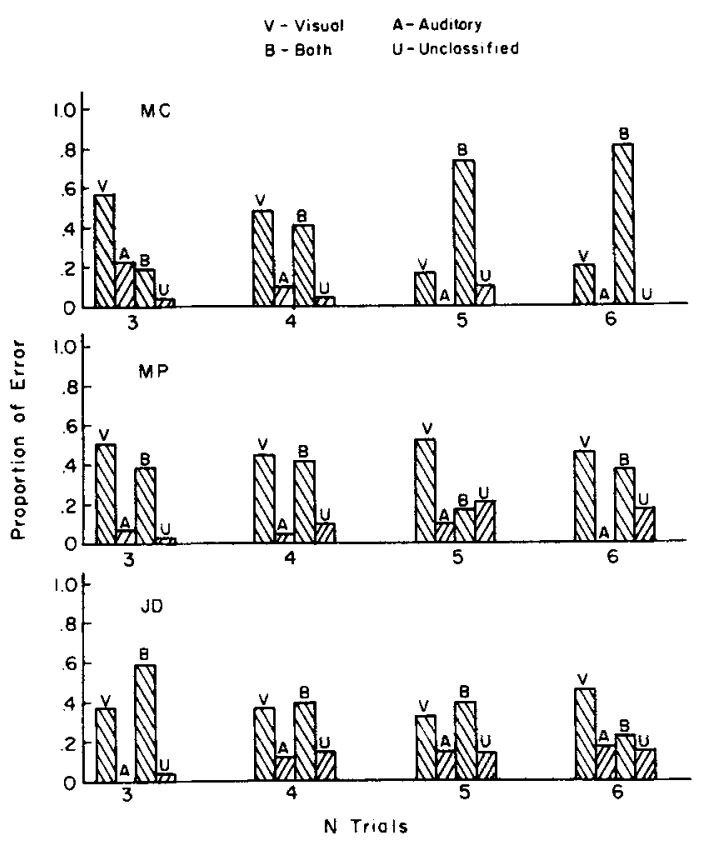

Fig. 4. Proportion of error by type of error corrected for a priori probability of making a given type of error, for each of three Ss, over four experimental conditions. Error types are verbal (V), auditory $(A)$, both verbal or auditory $(B)$, and unclassified $(U)$. 
Equally as important, the error content analysis (Fig. 4) suggests that when $S$ misnames a letter he does so primarily by confusing it with one that looks like it rather than with one that sounds like it. On the surface this appears to be at variance with predictions from models holding that visual information is auditorily encoded and maintained by an active rehearsal in an auditory information storage. (This refers to the models primarily of Sperling, 1963, and 1966; although the same principles are found in the models of Broadbent, 1958; and Melton, 1963.) However, these theorists are referring to situations in which the memory load is high, so that the perceiver would necessarily have to rehearse to maintain the information in memory until such time as he has to recall or recognize it. In this experiment, while $\mathbf{S}$ does have to make an oral report, he has only to maintain one letter in memory, and it seems quite unlikely that he would forget it or have it suffer interference during rehearsal from acoustically similar items. Thus, it is not surprising that auditorily confusable letters do not show up in error reports. If an error was made, it was because the letter was not seen clearly or completely.

A failure to manipulate memory load or to take it into account when predicting either auditory or visual errors is to miss the crux of the purpose of an auditory information storage and rehearsai process. Kaplan, Yonas, and Shurcliff (1966) attacked models stressing auditory information storages because they found visual errors in a task with low memory loads. However, it seems that, as in this experiment, only visual errors should be found when memory loads are low. Admittedly, much more work is needed on the relative properties of a short term visual storage and an auditory information storage in order to avoid these kinds of theoretical confusions.

Finally, it should be noted that effect of repetition when duration is held constant is quite similar to that found in the Haber and Hillman (1966) data. In that study, only a duration at threshold was used, which corresponds to the four-trial condition of this experiment. In the Haber and Hershenson (1965) experiment, little effect of repetition was found for seven-letter words if the duration is set well below threshold. The same seems to be true for single letters. However, setting the duration even slightly above threshold (around $1 \mathrm{msec}$. above for each of the three Ss) leads to appreciably better performance.

In summary then, a second attempt to demonstrate a reciprocity between duration and repetition in a recognition task failed. Increasing duration even by small amounts always swamps the effects of further repetition, such that one long flash always results in greater clarity of the stimulus than two or more shorter flashes summing to the same duration. Some implications of this finding were explored with respect to information processing of visual stimulation, and to auditory as compared to visual storage processes.

\section{References}

Boynton, R. M. Temporal processes in vision. In W. A. Rosenblith (Ed.), Sensory communication. Cambridge: MIT Press, 1961. Pp. 739-756.

Broadbent, D. E. Perception and communication. London: Pergamon Press, 1958. Pp. 297-301.

Eriksen, C. W., \& Hoffman, M. Form recognition at brief durations as a function of adapting field and interval between stimulations. J. exp. Psychol., 1963, 66, 485-499.

Gilinsky, A. S. The span and the scale: a bridge between attention and memory. IBM Res. Rep., RC1214, 1964, 1-64.

Haber, R. N. The effect of prior knowledge of the stimulus on word recognition processes. J. exp. Psychol, 1965, 69, 282-286.

Haber, R. N. Repetition as a determinant of perceptual recognition processes. In J. C. Mott-Smith, W. Wather-Dunn, H. Blum, and P. Lieberman (Eds.), Symposium on models for the perception of speech and visual form. Cambridge: MIT Press, 1966a (in press).

Haber, R. N. Perceptual recognition: the direct effect of repetition, duration, frequency and prior $k$ nowledge. Paper presented at the Psychonomic Society Convention, St. Louis, 1966b (in preparation for publication).

Haber, R. N. Perceptual processes and general cognitive activity. In J. F. Voss (Ed.), Approaches to thought. Pittsburgh: University of Pittsburgh Press, 1967 (in press).

Haber, R. N., \& Hershenson, M. The effects of repeated brief exposures on the growth of a percept. J. exp. Psychol., 1965, $69,40-46$.

Haber, R. N., \& Hillman, E. R. Changes in single letter clarity with repetition. Percept. \& Psychophys., 1966, 1, 347-350.

Haber, R. N., \& Meiselman, C. H. Estimates of false alarm reports in studies of perceptual recognition. Paper in preparation, 1967.

Hershenson, M. Perceptual microgenesis as a function of "word" structure. Paper presented at Midwestern Psychological Association Meeting, 1965.

Hershenson, M., Haber, R. N. The role of meaning in the perception of briefly presented words. Canad. J. Psychol., 1965, $19,42-46$.

Kaplan, G. A., Yonas, A., \& Shurcliff, A. Visual and acoustic confusability in a visual search task. Percept. \& Psychophys., $1966,1,172-174$.

Mayzner, M, S., \& Schoenberg, K. M. Short term retention and presentation rate. Psychon. Sci., 1965, 2, 111-112.

Melton, A. W. Implications of short term memory for a general theory of memory. J. verbal Learn. verbal Behav., 1963, 2, 1-21.

Pylyshyn, $Z$. $W$. Information available from two consecutive exposures of visual displays. Canad. J. Psychol., 1965, 19, 133144.

Smith, F., \& Carey, P. Temporal factors in visual information processing. Canad. J. Psychol., 1966, 20, 337-342.

Sperling, G. The information available in brief visual presentations. Psychol. Monogr., 1960, 74, Whole Number 498.

Sperling, G. A model for visual memory tasks. Hum. Factors, 1963, 5, 19-31.

Sperling, G. Successive approximations to a model for short term memory. Proc. 18th Int. Cong. Psychol., Moscow, 1966. Amsterdam: North Holland Publishing Company, 1967, (in press).

\section{Note}

1. This research was supported in part by grants from the National Science Foundation, GB-2909, and the United States Public Heaith Service, MH-10753, to the second author.

(Accepted for publication January 9, 1967.) 